\section{Detection of Quinolones Resistance in Ureaplasma urealyticum Clinical Isolates}

\section{Abstract}

Background: Widespread use of quinolones are increasing resistance to these antibiotic in Ureaplasma urealyticum. Quinolone resistance occur in U. urealyticum due to Point mutations in DNA topoisomerase and DNA gyrase genes (gyrA, gyrB, parC and parE). The aim of this study were determination of point mutation in clinical isolate by PCR and sequencing methods.

Materials and Methods: To investigate the prevalence of quinolone resistance mutations, $30 \mathrm{U}$. urealyticum positive sample were gathered from pregnant women, referred to obstetrics and gynecology section or prenatal clinic in Beasat Hospital, Sanandaj, Iran. DNA extraction were performed. Point mutation of target genes were done after PCR amplification reaction by sequencing.

Results: The results of gene sequencing showed that the substitution of amino acids in codon 83 parC happened in 5 samples. Aspartic acid 82 Asparagine change caused by amino acid substitution $D$ to $N$ happened in 4 cases. The results of the gyrA gene sequencing showed that the amino acid substitution in codon 104 occurred in 2 sample. GUL104LYS amino acid substitution change occurred in 5 samples.

Conclusion: Quinolones are most common antibiotics effective in treatment infections caused by a $U$. urealyticum. Therefore early detection of resistance genes is essential to correct treatment regime to prevent the spread of resistant strains.

Keywords: U. urealyticum; Quinolones; gyrA; gyrB; parC; parE

\section{Rashid $\mathbf{R}^{1,2 *}$ and Amir safari ${ }^{2,3}$}

1 Cellular \& Molecular Research Center, Kurdistan University of Medical Sciences, Sanandaj, Iran

2 Microbiology department, Faculty of Medicine, Kurdistan University of Medical Sciences, Sanandaj, Iran

3 Student Research Committee, Kurdistan University of Medical Sciences, Sanandaj, Iran

\section{*Corresponding author:}

Rashid Ramazanzadeh

\section{Rashid@muk.ac.ir}

Kurdistan University of Medical Sciences, Sanandaj, Iran.

Tel: +989143104424

Citation: Rashid R, Safari A (2018) Detection of Quinolones Resistance in Ureaplasma urealyticum Clinical Isolates. Arch Clin Microbiol. Vol.9 No.3:82

Received: April 22, 2018; Accepted: May 11, 2018; Published: MaY 16, 2018

\section{Introduction}

Ureaplasma spp. are one of the sexually transmitted pathogens that considered etiology agent of urethritis, prostatitis, bacterial vaginosis, cervicitis and pelvic might be led to infertility in men and women [1-4]. For treatment of infections caused by this bacteria fluoroquinolones are most used antibiotics. This antibiotics killing Ureaplasma through the inhibition of DNA replication. Nowadays there are increasing report on acquired resistance to quinolones [5]. The mechanism of fluoroquinolone resistance in Ureaplasma are depend on point mutations in DNA gyrase and topoisomerase IV [4-7].

In order to figure out the resistance rate of $U$. urealyticum, it is necessary to analyze the mutation rates. Therefore, we have determined the mutation rate of $U$. urealyticum to quinolone antibiotics by using PCR and sequencing of GyrA and GyrB subunits of DNA gyrase and ParC, ParE subunits of topoisomerase IV in clinical isolates.

\section{Materials and Methods}

\section{Bacterial isolates}

This study was done on sample has been described previously [2]. Samples were gathered from pregnant women, referred to obstetrics and gynecology section or prenatal clinic in Beasat Hospital, Sanandaj, Iran. For all women endocervical samples were collected using a cotton swap into sterile $15-\mathrm{mL}$ falcon tubes containing $5 \mathrm{~mL}$ of phosphate-buffered saline and placed at $70^{\circ} \mathrm{C}$ until DNA extraction. 
DNA extraction

Samples were transferred to laboratory in standard condition. DNA extraction were done by Kit instruction (High pure PCR Template Preparation; Roche, Germany). Extracted DNA were stoked $-70^{\circ} \mathrm{C}$ until PCR test.

\section{PCR test}

PCR was performed in a final volume of $20 \mathrm{ml}$ for ParC and parE and gyrA genes with primers as shown in Table 1.

The PCR were done in a Thermocycler (Eppendorf, Hamburg, Germany) with program including: initial denaturation $94^{\circ} \mathrm{C}$ for 10 minutes, followed by 30 cycles of denaturation at $94^{\circ} \mathrm{C}$ for 1 minute, annealing at $64^{\circ} \mathrm{C}$ for 30 seconds, and extension at $72^{\circ} \mathrm{C}$ for 1 minute, and final extension at $72^{\circ} \mathrm{C}$ for 10 minutes. In order to observe the amplified PCR products, gel electrophoresis were accomplished in $1.5 \%$ gel agarose stained by ethidium bromide, and then photographed after visualized by UV light.

\section{Sequencing}

PCR products were sent to Sina Clone co for sequencing. Results of sequencing were analyzed Using Chromas pro V 2.1.1 software. Using online software including FASTA and European Bioinformatics Institute (EMBL-EBI) data were Aligned and point mutation detected.

\section{Result}

The study carried out, to investigate the prevalence of quinolone resistance mutations in 30 samples for parC, parE and gyrA genes as shown in Table 2. The results of gene sequencing showed that the substitution of amino acids in codon 83 parC happened. Ser83luc amino acid substitution caused by movement $S$ to $L$ in 5 samples. Aspartic acid 82 Asparagine change caused by amino acid substitution $D$ to $N$ happened in 4 cases. The results of the gyrA gene sequencing showed that the amino acid substitution in codon 104 occurred in 2 sample. GUL104LYS amino acid substitution change occurred in 5 samples.

Table 1 Primers were for PCR amplifications in clinical samples.

\begin{tabular}{|c|c|c|}
\hline $5^{\prime}$ to $\mathbf{3}^{\prime}$ sequence & primers & Gene \\
\hline TTGATGGTGATAGTGCAG & gyrA-F & gyrA \\
\hline TAGTAAGTCAGTGTGGGT & gyrA-R & \\
\hline CCCGTTCAACGACGTATTTT & ParC-F & parC \\
\hline TCTGTATAACGCATCGCAGC & ParC-R & \\
\hline TCGCAAACGTCCTGGAATGT & ParE-F & parE \\
\hline AGCGTTTTCATGCACACCAC & ParE-R & \\
\hline
\end{tabular}

Table 2 Point mutations results in studied samples by sequencing.

\begin{tabular}{|c|c|c|c|}
\hline \multicolumn{4}{|c|}{ Mutation } \\
\hline Genes & No & Yes & Total \\
\hline GyrA & $28(93 \%)$ & $2(7 \%)$ & 30 \\
\hline ParC & $24(40 \%)$ & $6(60 \%)$ & 30 \\
\hline Par E & $24(40 \%)$ & $6(60 \%)$ & 30 \\
\hline
\end{tabular}

\section{Discussion}

Many pathogenic bacteria that infect the genital tract of women, including genital mycoplasmas, Neisseria gonorrhoeae, Chlamydia trachomatis, Enterobacteriaceae, Gram-positive cocci, and Gardenella vaginalis. Mycoplasma families can cause chronic and subclinical genital infections that may have a negative impact on female fertility [1, 8-10]. Ureaplasma is one of the main causes of Non gonococcal urethritis (NGU) in men, also in pregnant and non-pregnant women causing premature delivery, spontaneous abortion, premature birth, vaginitis and cervicitis. Today, drug resistance is a major problem in most countries. This resistance is steadily rising due to the limited number of effective drugs, also is a threat in the control program [5,11-15].

DNA topoisomerase IV has two subunit that encoded by parC, and parE genes. Amino acids substitution such as D82N, S83I, and $\mathrm{E} 87 \mathrm{~L}$ in parC gene causes resistance to quinolones. There are also other mutations in the gene parC amino acid asparagine to aspartic acid is substituted in Area 82 and Area 87 is glutamic acid to leucine [16-19]. In our study the mutation frequency in resistance gene parC was $20 \%$.

Kawai in 2015 a study $U$. urealyticum, out of 158 isolates, $23.4 \%$ quinolone resistance in parC gene mutation that leads to changes in gene found a specific area called s83। [7]. parE gene is necessary for bacterial chromosome partitioning. The amino acid sequence encoded by the gene parE and parC have many similarities. Mutations in the $\mathrm{C}$-terminal amino acids encoded by this gene causes resistance to quinolones. A quinolone resistance in $U$. urealyticum, can occurred due to amino acids replacement in ASP435 to Asn.

DNA gyrase encoded by gyrA and gyrB genes. Replacement of amino acids Q104K in gyrA gene causes resistance to quinolones. In gyrA gene in the 104 position lysine is replaced by glutamine $[7,13,16,19-21]$. In our study the mutation rate in resistance gene gyrA was $6.6 \%$.

\section{Conclusion}

Quinolones are most common antibiotics effective in treatment infections caused by a $U$. urealyticum. Therefore early detection of resistance genes is essential to correct treatment regime to prevent the spread of resistant strains. Study also showed us parC gene mutation at position 82 and in position 104 of gyrA gene as a marker for resistance to quinolones in isolated $U$. urealyticum.

\section{List of Abbreviations}

Ureaplasma urealyticum(U. urealyticum), DNA gyrase genes (gyrA, gyrB, parC and parE).

\section{Availability of Data and Materials}

All data generated or analyzed during this study are included in this published article.

\section{Competing Interests}

The authors declare that there are no conflicts of interest regarding the publication of this article. 


\section{Funding}

This study is funded by Kurdistan University of Medical Sciences. The funding body managed the reviewing proposal of this study and they did not have any role in data collection, analysis, and interpretation of data and in writing this manuscript.

\section{Authors' Contributions}

Amir Safari carried out the study and collected data. Rashid Ramazanzadeh supervised the study, participated in designing

\section{References}

1 Ikonomidis A, Venetis C, Georgantzis D, Giaslakiotis V, Kolovos V, et al. (2016) Prevalence of Chlamydia trachomatis, Ureaplasma spp., Mycoplasma genitalium and Mycoplasma hominis among outpatients in central Greece: absence of tetracycline resistance gene tet(M) over a 4-year period study. New microbes and new infections 9: 8-10.

2 Ramazanzadeh R, Khodabandehloo M, Farhadifar F, Rouhi S, Ahmad A, et al. (2016) A Case-control Study on the Relationship between Mycoplasma genitalium Infection in Women with Normal Pregnancy and Spontaneous Abortion using Polymerase Chain Reaction. Osong public health and research perspectives 7: 334-338.

3 Farhadifar F, Khodabandehloo M, Ramazanzadeh R, Rouhi S, Ahmadi A, et al. (2016) Survey on association between Mycoplasma hominis endocervical infection and spontaneous abortion using Polymerase Chain Reaction. Int J Reprod Biomed (Yazd) 14: 181-186.

4 Mousavi A, Farhadifar F, Mirnejad R, Ramazanzadeh R (2014) Detection of genital mycoplasmal infections among infertile females by multiplex PCR. Iranian journal of microbiology 6: 398-403.

5 Xiao L, Crabb DM, Duffy LB, Paralanov V, Glass JI, et al. (2012) Chromosomal mutations responsible for fluoroquinolone resistance in Ureaplasma species in the United States. Antimicrobial agents and chemotherapy 56: $2780-2783$.

6 Schneider SC, Tinguely R, Droz S, Hilty M, Dona V, et al. (2015) Antibiotic Susceptibility and Sequence Type Distribution of Ureaplasma Species Isolated from Genital Samples in Switzerland. Antimicrobial agents and chemotherapy 59: 6026-6031.

7 Kawai $Y$, Nakura $Y$, Wakimoto T, Nomiyama M, Tokuda T, et al. (2015) In vitro activity of five quinolones and analysis of the quinolone resistance-determining regions of gyrA, gyrB, parC, and parE in Ureaplasma parvum and Ureaplasma urealyticum clinical isolates from perinatal patients in Japan. Antimicrobial agents and chemotherapy 59: 2358-2364.

8 Song T, Ye A, Xie X, Huang J, Ruan Z, et al. (2014) Epidemiological investigation and antimicrobial susceptibility analysis of ureaplasma species and Mycoplasma hominis in outpatients with genital manifestations. Journal of clinical pathology 67: 817-820.

9 Hunjak B, Sabol I, Vojnovic G, Fistonic I, Erceg AB, et al. (2014) Ureaplasma urealyticum and Ureaplasma parvum in women of reproductive age. Archives of gynecology and obstetrics 289: 407-412.

10 Ahmadi A, Khodabandehloo M, Ramazanzadeh R, Farhadifar $F$ Nikkhoo B, et al. (2014) Association between Ureaplasma urealyticum endocervical infection and spontaneous abortion. Iranian journal of microbiology 6: 392.

11 Beeton ML, Chalker VJ, Jones LC, Maxwell NC, Spiller OB (2015) Antibiotic Resistance among Clinical Ureaplasma Isolates Recovered and conducting it, and prepared the original version of the manuscript. All authors studied and approved the content of the present manuscript and participated in revising the paper.

\section{Acknowledgement}

This is part of Amir Safari (MSc in Medical Microbiology) thesis. The authors wish to extend their gratitude to the Research Deputy of the Kurdistan University of Medical Sciences for financial support.

from Neonates in England and Wales between 2007 and 2013. Antimicrobial agents and chemotherapy 60: 52-56.

12 Kotrotsiou T, Exindari M, Diza E, Gioula G, Melidou A, et al. (2013) Prevalence and antimicrobial susceptibility of Ureaplasma urealyticum in asymptomatic women in Northern Greece. Hippokratia 17: 319-321.

13 Kamiya Y, Shimada Y, Ito S, Kikuchi M, Yasuda M, et al. (2013) Analysis of the quinolone-resistance determining region of the gyrA gene and the analogous region of the parC gene in Ureaplasma parvum and Ureaplasma urealyticum detected in first-void urine of men with nongonococcal urethritis. The Journal of antimicrobial chemotherapy 68: $480-482$.

14 Dhawan B, Malhotra N, Sreenivas V, Rawre J, Khanna N, et al. (2012) Ureaplasma serovars \& their antimicrobial susceptibility in patients of infertility \& genital tract infections. The Indian journal of medical research 136: 991-996.

15 Ventolini G, Lee M (2011) Clinical antibiotic response of mycoplasma and ureaplasma in patients with symptomatic recurrent vulvovaginal colonization. The Journal of reproductive medicine 56: 138-141.

16 Piccinelli G, Gargiulo F, Biscaro V, Caccuri F, Caruso A, et al. (2016) Analysis of mutations in DNA gyrase and topoisomerase IV of Ureaplasma urealyticum and Ureaplasma parvum serovars resistant to fluoroquinolones. Infection, genetics and evolution : journal of molecular epidemiology and evolutionary genetics in infectious diseases 47: 64-67.

17 Song J, Qiao Y, Kong Y, Ruan Z, Huang J, et al. (2015) Frequent topoisomerase IV mutations associated with fluoroquinolone resistance in Ureaplasma species. Journal of medical microbiology 64: 1315-1320.

18 Yamaguchi Y, Takei M, Kishii R, Yasuda M, Deguchi T (2013) Contribution of topoisomerase IV mutation to quinolone resistance in Mycoplasma genitalium. Antimicrobial agents and chemotherapy 57: $1772-1776$.

19 Bebear CM, Renaudin H, Charron A, Clerc M, Pereyre S, et al. (2003) DNA gyrase and topoisomerase IV mutations in clinical isolates of Ureaplasma spp. and Mycoplasma hominis resistant to fluoroquinolones. Antimicrobial agents and chemotherapy 47: 3323-3325.

20 Beeton ML, Chalker VJ, Kotecha S, Spiller OB (2009) Comparison of full gyrA, gyrB, parC and parE gene sequences between all Ureaplasma parvum and Ureaplasma urealyticum serovars to separate true fluoroquinolone antibiotic resistance mutations from non-resistance polymorphism. The Journal of antimicrobial chemotherapy 64: 529-538.

21 Reinhardt AK, Bebear CM, Kobisch M, Kempf I, Gautier-Bouchardon AV (2002) Characterization of mutations in DNA gyrase and topoisomerase IV Involved in quinolone resistance of Mycoplasma gallisepticum mutants obtained in vitro. Antimicrobial agents and chemotherapy 46: 590-593. 